\title{
Kariesdiagnostik mittels künstlicher Intelligenz: Zukunftsmusik oder Realität?
}

\author{
Falk Schwendicke, Joachim Krois
}

\section{Durch die Anwendung von maschinellem Sehen und maschinellem Lernen haben KI-Applikationen für die Kariesdiagnostik großes Potenzial.}

\section{Einführung}

Die Detektion kariöser Läsionen ist täglicher Bestandteil der zahnärztlichen Praxis; in Deutschland werden jährlich vermutlich 50-100 Millionen kariöse Läsionen versorgt [1]. Seit über 100 Jahren stehen diverse diagnostische Verfahren für die Erkennung von Karies zur Verfügung. Der vorliegende Artikel beleuchtet eine völlig neue Methode zur Unterstützung in der Kariesdiagnostik; den Einsatz von künstlicher Intelligenz (KI). Wir werden aufzeigen, warum KI für den Zahnarzt im Rahmen der täglichen Kariesbefundung hilfreich sein kann, wo aktuelle KI-Anwendungen hinsichtlich ihrer Genauigkeit und Generalisierbarkeit stehen und abschließend aufzeigen, dass KI für die Kariesdiagnostik heute nicht mehr Zukunftsmusik, sondern Realität ist.

\section{Kariesdiagnostik}

Über einen Großteil der letzten 100 Jahre hat sich Kariesdiagnostik vor allem auf das Erkennen von kavitierten kariösen Läsionen fokussiert. Dies basierte auf einem vor allem restaurativ geprägten Verständnis der Kariestherapie (Karies sollte vollständig entfernt werden, um die Erkrankung zu heilen; Infektionshypothese), aber auch auf der extrem weiten Verbreitung kavitäter kariöser Läsionen (epidemiologische Krankheitslast). Zudem standen Zahnärzten vor allem restaurative Therapiestrategien (u.a. Amalgamfüllungen) zur Verfügung. Eine rein visuell taktile Karieserkennung (unter Einsatz unserer Augen und einer Sonde) erschien zweckmäßig und ausreichend; können eingebrochene größere Kariesläsion auf diesem Wege doch zuverlässig erkannt - also weder übersehen (Sensitivität) noch fälschlicherweise gesunde Flächen als Karies erkannt (Spezifität) werden [2].

Neue wissenschaftliche Erkenntnisse haben in der Kariesdiagnostik neue Wege aufgezeigt. Das Verständnis von Karies als eine kontrollierbare Erkrankung, die in frühen Stadien kausal (also nicht restaurativ) therapiert werden kann, und die resultierende Möglichkeit, frühe kariöse Läsionen heilen oder zumindest arretieren zu können, führ- ten zu einem Umdenken auch in der Kariesdiagnostik. Der Detektion früher (nicht kavitierter) Läsionen auf Glattflächen, Approximalflächen oder im Fissurenbereich kommt heute eine große Bedeutung zu. Nur wenn frühe kariöse Läsionen rechtzeitig identifiziert und einer adäquaten Therapie, z. B. noninvasiv mittels Fluoriden oder mikroinvasiv mittels Versiegelung oder Infiltration, zugeführt werden, gelingt deren Heilung oder Arretierung. Sind Läsionen erst einmal kavitiert, so besteht Konsens, dass in vielen Fällen restaurativ therapiert werden muss, um Oberflächenintegrität, Funktionalität und Ästhetik des Zahns wiederherzustellen [2]. Zudem hat sich auch die epidemiologische Krankheitslast deutlich verändert; kavitierte Läsionen sind heute deutlich seltener als noch vor 30 Jahren, während die Zahl von nicht kavitierten frühen Läsionen deutlich zugenommen hat [3]. Dies ist vor allem auf individuelle und professionelle Präventionsbemühungen, beispielsweise den Einsatz fluorierter Zahnpasta, zurückzuführen.

Die frühe Detektion von Karies eröffnet also neue therapeutische Optionen. Es besteht zudem der epidemiologisch hergeleitete Bedarf einer frühen Kariesdiagnostik. Schlussendlich haben wir heute auch die technischen Möglichkeiten, um frühe Läsionen aufzufinden und erfolgreich behandeln zu können. Einerseits hat sich aus diesen Überlegungen heraus die visuell-taktile Kariesdetektion gewandelt; es werden nicht mehr nur kavitierte Läsionen detektiert, sondern auch frühe Karies, z. B. entlang der sogenannten ICDAS-Klassen (International Caries Detection and Assessment System). Hierbei werden beispielsweise frühe, nur nach Trocknung detektierbare Läsionen von klar und auch ohne Trocknung erkennbaren nicht kavitierten sowie Läsionen mit Schmelz- oder Dentineinbruch unterschieden [4]. Andererseits kommen zunehmend Hilfsmittel zum Einsatz, um kariöse Läsionen an weniger zugänglichen Stellen (z. B. Approximalflächen in geschlossenen Zahnreihen) detektieren und generell über mehrere Untersuchungen hinweg regelmäßig kontrollieren zu können (Monitoring). Im Folgenden werden wir detaillierter auf die röntgenologische Kariesdiagnostik eingehen. 


\section{Bissflügelröntgen: Noch immer der Standard}

Zur Röntgendiagnostik von Karies kommen vor allem Bissflügelaufnahmen zum Einsatz. Diese sollten risikoadjustiert vor allem bei nicht einsehbaren Approximalräumen regelmäßig, also alle 12-36 Monate, angefertigt werden. Vor allem die Detektion approximaler früher Läsionen ist auf Bissflügelaufnahmen besser möglich als visuell-taktil, und alternative (röntgenstrahlungsfreie) Detektionsmethoden (z.B. laserinduzierte Fluoreszenz, Nahinfrarottransillumination) sind dem Bissflügelröntgen noch immer teilweise deutlich unterlegen (sie bringen jedoch zahlreiche andere Vorteile mit sich und sind deshalb zunehmend sinnvoll einsetzbar) [5-7].

Die Kariesdiagnostik auf solchen Röntgenaufnahmen ist für Zahnärzte allerdings eine Herausforderung. Eine systematische Übersichtsarbeit aus dem Jahr 2015 hat 117 Studien, die Karies auf insgesamt fast 20000 Zahnflächen untersucht haben, eingeschlossen und eindrucksvoll nachgewiesen, dass Zahnärzte gut in der Lage sind, auf Bissflügelaufnahmen gesunde Flächen auch als gesund zu erkennen (hohe Spezifität, im Mittel 70-97\%), jedoch oftmals nicht alle kariösen Flächen auffinden, also eine geringe Sensitivität aufweisen (im Mittel 24-56\%). Bei frühen kariösen Läsionen wurden 75\% durch Zahnärzte nicht detektiert [8]. Es kann argumentiert werden, dass bei einer langsam voranschreitenden Erkrankung wie Karies, bei der zudem ja Untersuchungen regelmäßig wiederholt werden, eine solche niedrige Sensitivität weniger dramatisch ist als eine niedrige Spezifität. Diverse Untersuchung unterstreichen dies auch; Karies zu übersehen verhindert zwar eine frühe Therapie und führt somit dazu, dass eine gewisse Zahl an Läsionen voranschreitet und schlussendlich restaurativ therapiert werden muss; das falsch positive Erkennen von Karies gefolgt von einer restaurativen Therapie ist jedoch deutlich schädlicher [9]. Die hohe Spezifität der Zahnärzte ist somit zu begrüßen. Nichtsdestotrotz ist es erstrebenswert, auch die Sensitivität zu erhöhen. In den letzten 20 Jahren sind diverse technische Ansätze zur Verbesserung der Karieserkennung auf Röntgenbildern getestet worden. Diese haben allerdings nur geringe Verbreitung in der Praxis gefunden, unter anderem weil ihre Genauigkeitsvorteile begrenzt waren.

Heute versprechen Anwendungen der KI, eben genau diese Genauigkeitsvorteile zu liefern. Erste KI-Anbieter aus Deutschland wie die aus der Charité - Universitätsmedizin Berlin ausgegründete dentalXrai $\mathrm{GmbH}$ werben zusammen mit ihren Vertriebspartnern mit dem Slogan, „nie wieder Karies [zu] übersehen“. Im Folgenden werden wir uns der technologischen Grundlage hinter solchen KIAnwendungen widmen und die Daten zu KI für die Kariesdiagnostik detailliert darlegen.

\section{Künstliche Intelligenz in der Diagnostik}

Der Begriff KI wurde Mitte der 1950er-Jahre geprägt und beschreibt Maschinen oder Systeme, die in der Lage sind, Aufgaben zu erfüllen, die (bisher) dem Menschen vorbehalten waren. Moderne KI-Ansätze bedienen sich vor allem des sogenannten maschinellen Lernens (ML). Beim ML programmieren nicht Menschen bestimmte Regeln, die es Computern dann erlauben, durch Ausführung dieser Regeln komplexe Aufgaben zu erfüllen; vielmehr lernen Maschinen selbst durch das Analysieren großer Datenmengen die notwendigen Regeln zur Erfüllung der Aufgabe. Im Alltag sind ML-Modelle bereits weit verbreitet und werden zur Gesichtserkennung im Mobiltelefon, in Überwachungskameras oder dem autonomen Fahren eingesetzt.

Bei dem in der Medizin üblichen „überwachten ML“ werden hierzu Tausende von Datenpunkten (z. B. Bilder) zusammen mit jeweils einer passenden Dateninformation (z. B.: Auf diesem Bild befindet sich Karies; auf diesem Bild befindet sich an Zahn 36 Karies; auf diesem Bild zeigen folgende Pixel Karies) zum Trainieren der notwendigen KI-Modelle eingesetzt. Die Modelle werden in zahlreichen Trainingsrunden (Epochen) iterativ verfeinert, bis schließlich das statistische Muster, welches hinter der Beziehung zwischen den Datenpunkten und den Dateninformationen liegt, ausreichend erkannt und abgebildet worden ist, und damit auch das trainierte Modell sinnstiftend eingesetzt werden kann. Dieses Modell kann dann auf neuen, bisher nicht im Trainingsdatensatz enthaltenen Daten getestet und angewandt werden ( $\bullet$ Abb. 1).

Eine Spezialform des ML ist das sogenannte tiefe Lernen (Deep Learning), in dem Künstliche Neuronale Netzwerke (KNN) trainiert werden, um komplexe Aufgaben wie Bild-, Text- oder Sprachanalysen durchzuführen [10]. Im Bereich des maschinellen Sehens (Bildanalyse) kommen vorrangig sogenannte Konvolutionale Neuronale Netzwerke zum Einsatz. Die Besonderheit dieser Netzwerkarchitektur ist, dass dem eigentlichen KNN eine Systemkomponente bestehend aus Bildfiltern und mathematischen Operationen vorangeschaltet ist. Innerhalb dieser Komponente tastet eine Vielzahl von Filtern das Bild systematisch ab und extrahiert dabei relevante Informationen wie z. B. Farben, Formen, Kanten, Texturen etc. Diese Filter werden auch hintereinander geschaltet und deren Ausgabe skaliert, sodass feine und kleinteilige, aber auch makroskopische Strukturen erfasst und in numerische Repräsentationen überführt werden können. Diese hochdimensionalen und für den Menschen nicht erfassbaren Repräsentationen des Eingangsbildes werden dem KNN übergeben. Das KNN wird iterativ so lange trainiert, bis ein solcher Eingangsdatensatz möglichst fehlerfrei einem Ergebnis (z. B. kariöse Läsion auf Zahn 46), das vorab von Experten bestätigt wurde, zugeordnet werden kann. Über Iteration und Optimierung des KNN lernen diese 


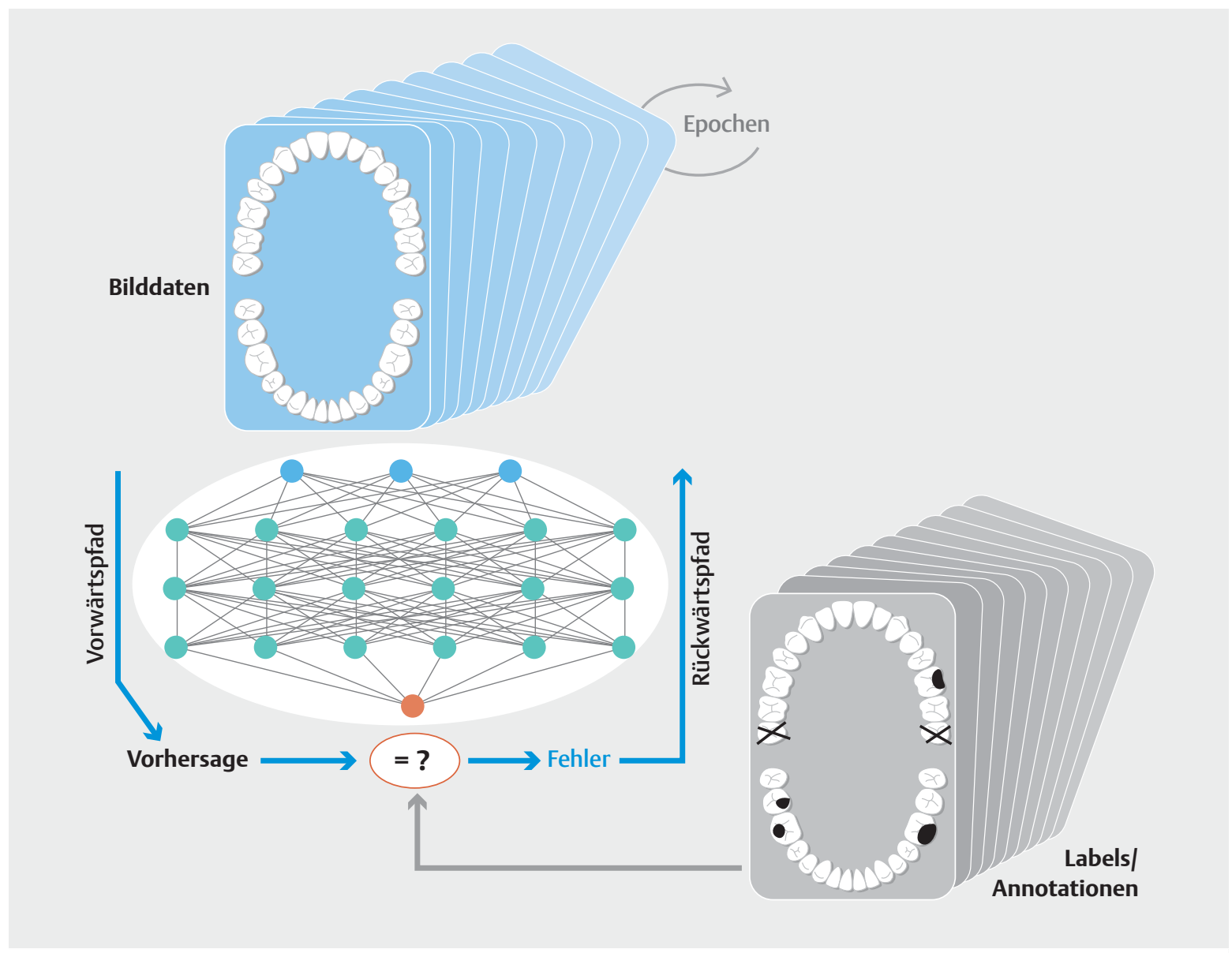

- Abb. 1 Maschinelles Lernen (ML). Beim „überwachten ML“ werden Datenpunkte durch ein Modell (hier ein Künstliches Neuronales Netzwerk, KNN) verarbeitet und das vorhergesagte Ergebnis mit jeweils einer passenden Dateninformation (hier von Experten markierte kariöse Stellen) abgeglichen. Falsche Vorhersagen führen dazu, dass die Modellparameter des KNN leicht angepasst werden, um bei der nächsten Iteration diesen Fehler nicht zu wiederholen. Ein auf ausreichend großen Datensätzen trainiertes Modell kann dann für neue, bisher nicht im Trainingsdatensatz enthaltenen Daten Vorhersagen treffen. Über Iteration und Optimierung des KNN lernen diese Modelle komplexeste statische Zusammenhänge und erreichen eine Leistungsfähigkeit, die der menschlichen Intelligenz ebenbürtig oder in bestimmten Fällen sogar überlegen sein kann.

Modelle komplexeste statische Zusammenhänge [11] in Bilddaten und erreichen eine diagnostische Leistungsfähigkeit, die der menschlichen Intelligenz ebenbürtig oder in bestimmten Fällen sogar überlegen sein kann [12].

\section{KI für Kariesdiagnostik}

In einer kürzlich publizierten Studie wurde eine KI-Anwendung zur Diagnostik von frühen und vorangeschrittenen kariösen Läsionen an Approximalflächen auf Bissflügelröntgenbildern evaluiert [13]. Die KI war an insgesamt ca. 3600 Röntgenbildern trainiert, validiert und getestet worden. Jedes Bild war von 3 unabhängigen zahnärztlichen Experten befundet worden (die Experten markierten die durch Karies betroffenen Pixel auf dem Bild) und anschließend von einem 4. Experten (Master-Reviewer) überprüft worden; dieser konnte die Gesamtheit der Be- funde überblicken, ergänzen oder löschen. Die Summe aller auf dem Bild eingezeichneten Pixel wurde schließlich als Referenztest (Goldstandard) zum Trainieren und Testen des Netzwerkes eingesetzt. Um Aussagen zur relativen Genauigkeit der KI im Vergleich mit Zahnärzten treffen zu können, wurden die Testdaten (140 Röntgenbilder) von weiteren 7 unabhängigen Zahnärzten befun$\operatorname{det}(\boldsymbol{A}$ Abb. 2).

Die Ergebnisse der Studie waren aufschlussreich: Die Spezifität von KI und Zahnärzten unterschied sich nicht signifikant und war insgesamt befriedigend hoch. Bei der Sensitivität war ein deutlicher Unterschied zwischen KI und Zahnärzten zu erkennen. Bei frühen, auf den Schmelz begrenzten Läsionen zeigte die KI eine bis zu 3-mal höhere Sensitivität als die Zahnärzte; nur ein Zahnarzt erreichte eine der KI ähnliche Sensitivität. Bei vorangeschrittenen Läsionen war der Unterschied zwischen KI und Zahn- 


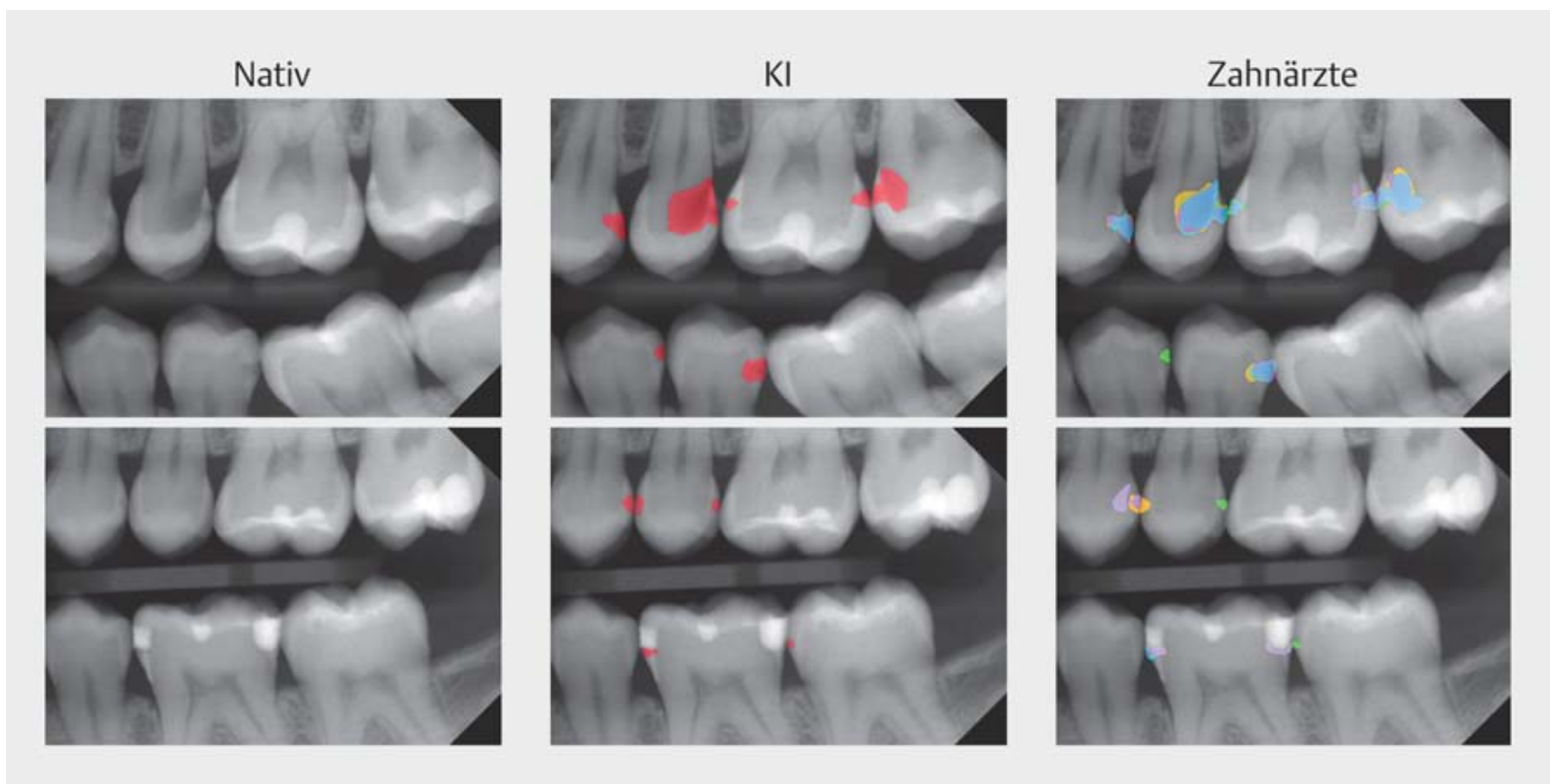

- Abb. 2 Kariesbefundung. Das KI-Modell (rot) und die Zahnärzte (rechts, verschiedene Farben) zeigen teils ähnliche, teils auch unterschiedliche Detektionsmuster. Die Zahnärzte weisen auch untereinander eine hohe Variabilität auf.

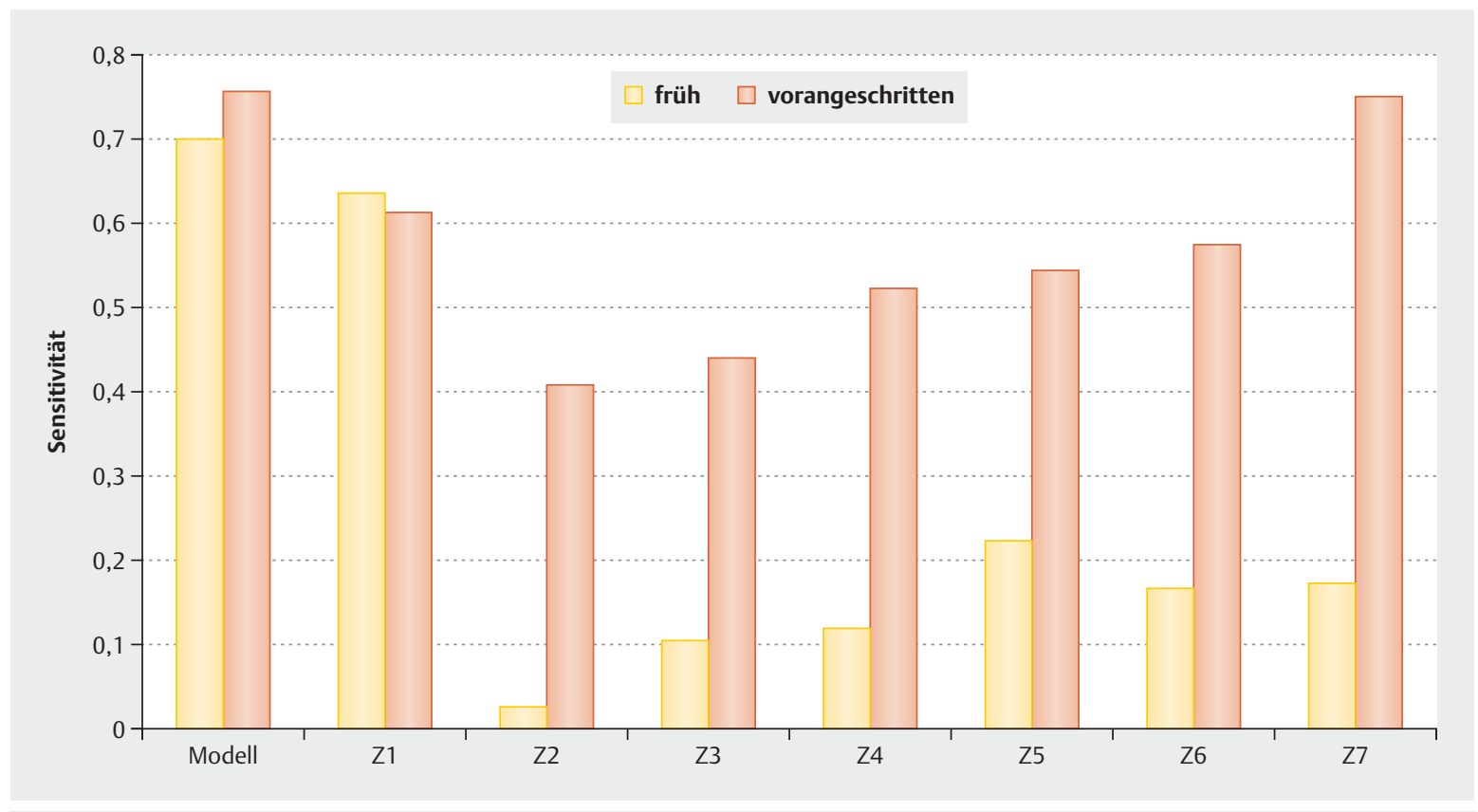

- Abb. 3 Sensitivität von KI und Zahnärzten (Z1-7) zur Erkennung von frühen und vorangeschrittenen Läsionen [13].

ärzten deutlich kleiner. Während also Zahnärzte deutliche Unterschiede in der Sensitivität je nach Läsionstiefe aufwiesen, war die KI sowohl für frühe als auch für vorangeschrittene Läsionen ähnlich sensitiv ( $\mathbf{A} \mathbf{b b}$. $\mathbf{3}$ ). Dies bestätigt einmal mehr den beschriebenen Unterschied zwischen dem maschinellen und menschlichen Sehen.
Die Daten dieser Studie wurden in einer 2. Untersuchung eingesetzt, um die Folgen einer unterschiedlichen Detektionsgenauigkeit mit und ohne KI-Unterstützung zu evaluieren [14]. Hierzu wurde ein Simulationsmodell eingesetzt. Dieses erlaubt, aus den Genauigkeitsdaten erwartete Therapiefolgen abzuleiten und dann wiederum die Therapien über die Lebenszeit eines Patienten abzubilden. Dies ist relevant, weil die meisten zahnärztlichen 


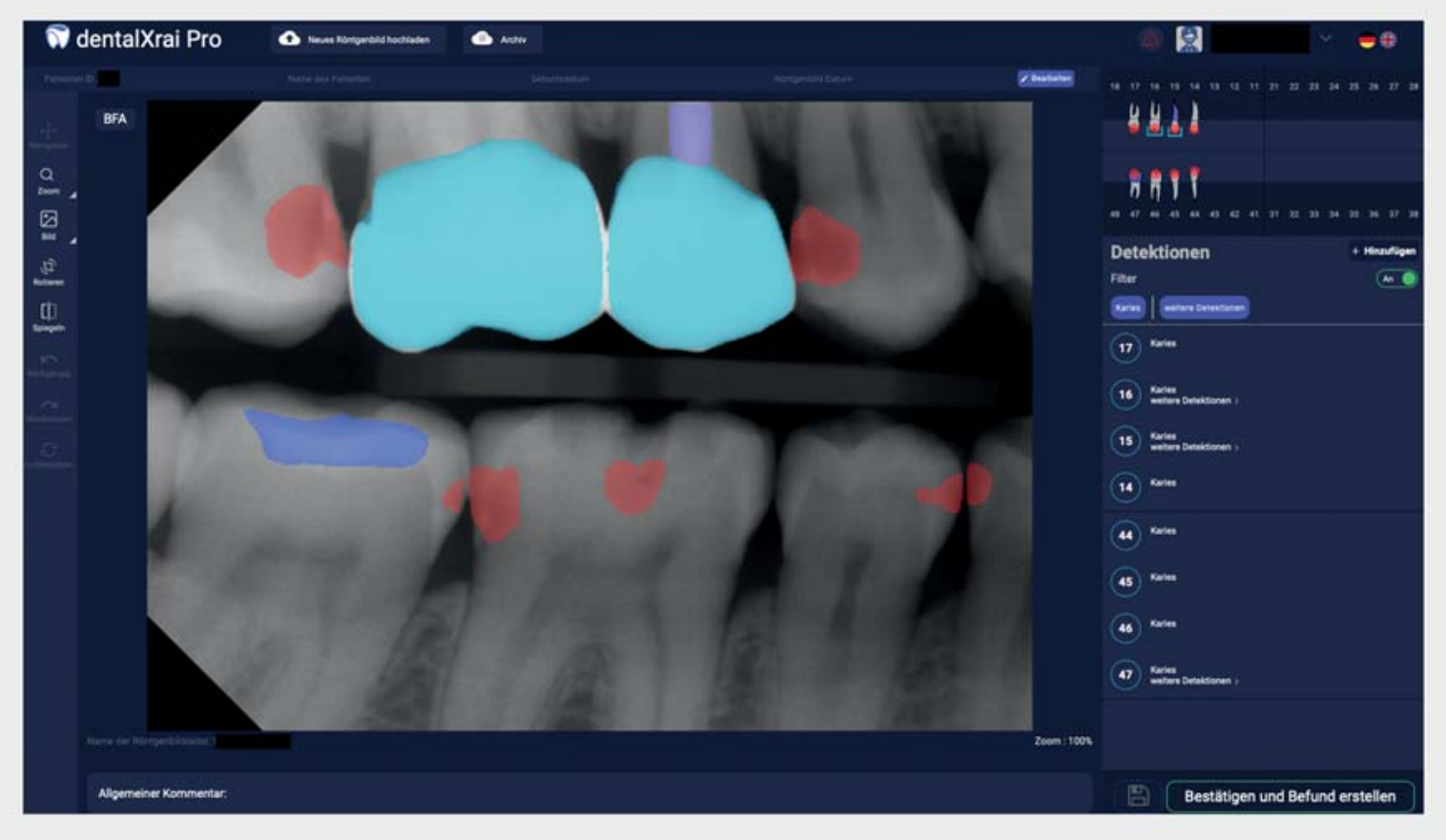

- Abb. 4 Das Diagnoseunterstützungssystem dentalXrai Pro. Ein zahnbasierter Vorbefund wird automatisch erstellt und grafisch angezeigt. Das native Röntgenbild und die farblich kodierten Detektionen (Karies in Rot, Kronen in Türkis, Füllungen in Blau) werden dargestellt. Eine Liste von Detektionen gibt einen detaillierten Überblick zum dentalen Status des Patienten. Interaktive Schaltelemete erlauben das schnelle Hinzu- und Wegschalten von farblich markierten Detektionen. Abschließend kann ein Bericht erstellt werden.

Therapien Langzeitwirkungen haben. Eine frühzeitig erkannte Läsion konnte in diesem Simulationsmodell beispielsweise mittels der Kariesinfiltration behandelt und in der überwiegenden Zahl der Fälle arretiert werden. Das Übersehen der Läsion führte mit einer bestimmten Wahrscheinlichkeit zu ihrem Voranschreiten und dem anschließenden Bedarf einer Restauration. Mit einer gewissen Wahrscheinlichkeit musste nun in den Folgejahren diese Restauration repariert oder ersetzt werden. Auch das Risiko einer notwendigen endodontischen oder weitergehenden restaurativen Behandlung (Überkronung) und sogar die Notwendigkeit einer Zahnextraktionen und von Zahnersatz wurden abgebildet.

Die Studie kam zu dem Schluss, dass die höhere Sensitivität des KI-Modells zur Erkennung früher Läsionen geeignet ist, sowie um die Notwendigkeit invasiver Folgetherapien zu reduzieren und Zähne langfristig bei geringeren Behandlungskosten zu erhalten. Dies galt jedoch nur, wenn frühe Läsionen adäquat - also nicht restaurativ behandelt wurden. In einem alternativen Simulationsszenario wurde angenommen, dass jede detektierte Läsion mit einer Füllung behandelt würde; in diesem Fall führt der Einsatz von KI zu Überbehandlung und höheren Kosten. Es wird demnach zentral sein, dass KI-Anwendung zur Kariesdiagnostik mittelfristig nicht nur das Vorhan- densein einer Karies anzeigen, sondern auch deren Tiefe und zur Verfügung stehende Therapieoptionen (Therapieempfehlungen).

Der Mehrwert von Kl-gestützten Anwendungen für den praktizierenden Zahnarzt geht aber über die Kariesdiagnostik weit hinaus. So können KNN auch für die Detektion weiterer Pathologien wie z. B. apikale Läsionen oder die Vermessung des parodontalen Knochenabbaus eingesetzt werden. Auch die Detektion und Klassifikation von Zähnen selbst oder verschiedener Restaurationen, wie Kronen, Füllungen, Implantaten etc. ist mit dieser Technologie zu bewerkstelligen. Führt man nun diese unterschiedlichen Funktionen in einem System zusammen, so entsteht ein Diagnoseunterstützungssystem, das einen diagnostischen Vorbefund allein basierend auf einem Bilddatensatz erstellen kann ( $\bullet$ Abb. 4 ). Diese Teilautomatisierung des diagnostischen Prozesses kann dem Zahnarzt Arbeit abnehmen und eine nützliche Ergänzung in der modernen digitalen zahnärztlichen Praxis sein. 


\section{SCHLUSSFOLGERUNGEN}

Die Detektion früher Läsionen ist ein zentrales Ziel moderner Kariesdiagnostik. Hierzu kommen zunehmend technische Hilfsmittel zum Einsatz; der Standard ist und bleibt das Bissflügelröntgen. Zahnärzte zeigen auf Bissflügelbildern eine hohe Spezifität (wenig falsch positive Befunde), aber eine eingeschränkte Sensitivität (viele übersehene Läsionen). Unter Anwendung von maschinellem Sehen und maschinellem Lernen haben KI-Applikationen für die Kariesdiagnostik großes Potenzial. Vorhandene Studien deuten darauf hin, dass KI gerade zur Erkennung früher Kariesläsionen den Zahnarzt unterstützen und zudem die Befund- und Reportqualität erhöhen kann bei gleichzeitiger Steigerung der Diagnostikeffizienz. Für den Gesundheitsnutzen der Patienten ist jedoch der sich anschließende Therapieentscheid zentral; frühe kariöse Läsionen sollten non- oder mikroinvasiv, nicht aber restaurativ behandelt werden. KI-basierte Röntgendiagnostik ist keine Zukunftsmusik, sie ist vielmehr heute schon Realität.

\section{Interessenkonflikt}

FS und JK sind Mitgründer eines Start-ups, das KI-Software zur Röntgenbildanalyse herstellt. Der vorliegende Artikel wurde unabhängig von diesem Start-up konzipiert und verfasst.

\section{Autorinnen/Autoren}

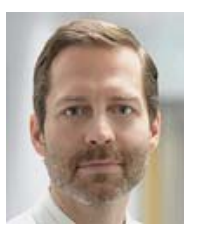

\section{Univ.-Prof. Dr. Falk Schwendicke}

Abteilung für Orale Diagnostik, Digitale Zahnheilkunde und Versorgungsforschung, Charité - Universitätsmedizin Berlin

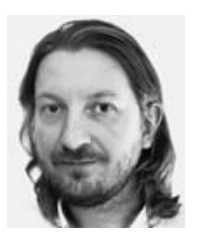

\section{Dr. Joachim Krois}

Abteilung für Orale Diagnostik, Digitale Zahnheilkunde und Versorgungsforschung, Charité - Universitätsmedizin Berlin

\section{Korrespondenzadresse}

Univ.-Prof. Dr. Falk Schwendicke, MDPH

Abteilung für Orale Diagnostik, Digitale Zahnheilkunde und Versorgungsforschung

CharitéCentrum 3 für Zahn-, Mund- und Kieferheilkunde

Charité - Universitätsmedizin Berlin

Aßmannshauser Straße 4-6

14197 Berlin

Deutschland

falk.schwendicke@charite.de

\section{Literatur}

[1] Schwendicke F, Krois ], Jordan R. Can We Predict Usage of Dental Services? An Analysis from Germany 2000 to 2015. JDR Clin Trans Res 2020; 5: 349-357

[2] Schwendicke F, Splieth C, Breschi L et al. When to intervene in the caries process? An expert Delphi consensus statement. Clin Oral Investig 2019; 23: 3691-3703

[3] Jordan AR, Krois J, Schiffner $U$ et al. Trends in caries experience in the permanent dentition in Germany 1997-2014, and projection to 2030: Morbidity shifts in an aging society. Sci Rep 2019; 9: 5534

[4] Ismail Al, Sohn W, Tellez M et al. The International Caries Detection and Assessment System (ICDAS): an integrated system for measuring dental caries. Community Dent Oral Epidemiol 2007; 35: 170-178

[5] Ortiz MIG, de Melo Alencar C, Freitas De Paula BL et al. Accuracy of near-infrared light transillumination (NILT) compared to bitewing radiograph for detection of interproximal caries in the permanent dentition: A systematic review and meta-analysis. J Dent 2020; 98: 103351

[6] Neuhaus KW, Ellwood R, Lussi A et al. Traditional lesion detection aids. Monogr Oral Sci 2009; 21: 42-51

[7] Gimenez T, Minatel Braga M, Procida Raggio D et al. Fluorescence-based methods for detecting caries lesions: systematic review, meta-analysis and sources of heterogeneity. PLoS One 2013; 8: e60421

[8] Schwendicke F, Tzschoppe M, Paris S. Radiographic caries detection: A systematic review and meta-analysis. J Dent 2015; 43: 924-933

[9] Schwendicke F, Paris S, Stolpe M. Detection and treatment of proximal caries lesions: Milieu-specific cost-effectiveness analysis. J Dent 2015; 43: 647-655

[10] LeCun Y, Bengio Y, Hinton G. Deep learning. Nature 2015; 521: 436-444

[11] Hornik K. Approximation capabilities of multilayer feedforward networks. Neural Networks 1991; 4: 251-257

[12] Esteva A, Robicquet A, Ramsundar B et al. A guide to deep learning in healthcare. Nat Med 2019; 25: 24-29

[13] Cantu AG, Gehrung S, Krois J et al. Detecting caries lesions of different radiographic extension on bitewings using deep learning. J Dent 2020; 100: 103425

[14] Schwendicke F, Rossi JG, Göstemeyer G et al. Cost-effectiveness of Artificial Intelligence for Proximal Caries Detection. J Dent Res 2020. doi:10.1177/0022034520972335

[15] Schwendicke F, Golla T, Dreher M et al. Convolutional neural networks for dental image diagnostics: A scoping review. J Dent 2019; 91: 103226

Bibliografie

ZWR - Das Deutsche Zahnärzteblatt 2021; 130: 99-104

DOI $\quad 10.1055 / \mathrm{a}-1389-3728$

ISSN 0044-166X

(C) 2021. Thieme. All rights reserved.

Georg Thieme Verlag KG, Rüdigerstraße 14,

70469 Stuttgart, Germany 